Document downloaded from:

http://hdl.handle.net/10251/164958

This paper must be cited as:

Llorens Molina, JA.; Vacas, S.; Castell-Zeising, V.; Verdeguer Sancho, MM. (2020). Seasonal variations of essential oils from five accessions of Mentha longifolia (L.) L. with selected chemical profiles. Journal of Essential Oil Research. 32(5):1-10.

https://doi.org/10.1080/10412905.2020.1773328

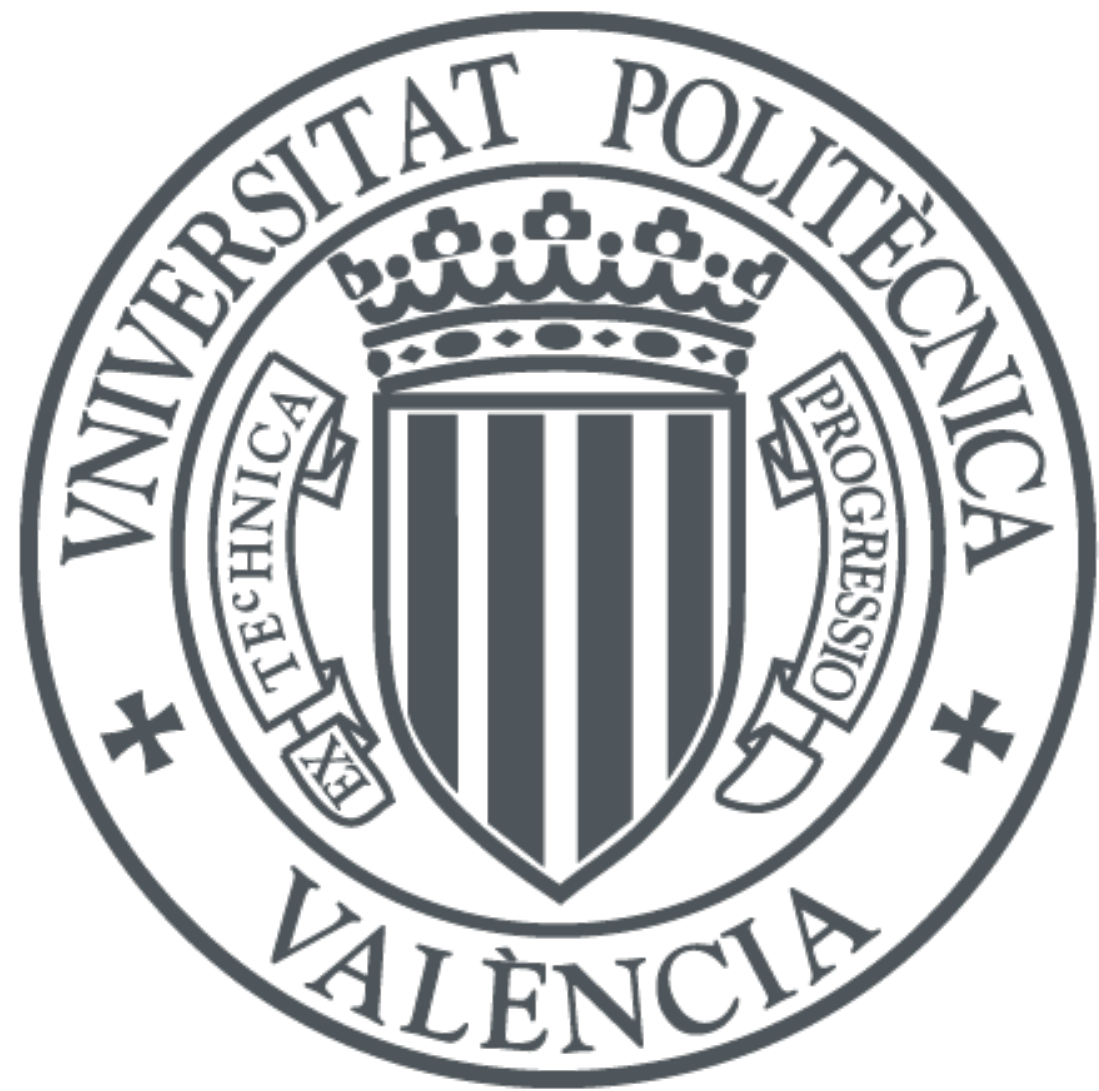

The final publication is available at

https://doi.org/10.1080/10412905.2020.1773328

Copyright Taylor \& Francis

Additional Information 


\title{
Seasonal variations of essential oils from five accessions of Mentha longifolia (L.) L. with selected chemical profiles
}

Llorens-Molina. Juan A. ${ }^{1}$; Vacas. S. ${ }^{2}$; Castell. V. ${ }^{3}$, Verdeguer, $\mathrm{M}^{1}$

${ }^{1}$ Mediterranean Agroforestry Institute. Universitat Politècnica de València. Camí de Vera. s/n. 46022. Valencia. SPAIN.

${ }^{2}$ Centre for Agricultural Chemical Ecology. (Mediterranean Agroforestry Institute). Universitat Politècnica de València.

${ }^{3}$ Department of Plant Production. School of Agricultural Engineering and Environment. Universitat Politècnica de València

*.iuallom2@qim.upv.es

\begin{abstract}
Essential oil (EO) yield and composition of five accessions of Mentha longifolia (L.) L. (leaves) were determined throughout their vegetative cycle by GC-MS and GC-FID analysis. These accessions were selected from individuals of a wild population located in Teruel (Spain). This selection was carried out based on the individual TLC profiles of dichloromethane extracts from leaves in such a way that each accession showed a homogeneous and characteristic composition. The accession rich in $\alpha$-terpineol acetate and carvone acetate has not been previously reported.
\end{abstract}

As for EO yield, all the accessions showed the maximum value in advanced flowering stage. With regard to composition, significant changes were noted in the accession A respect to the relative amounts of its main compounds, piperitone and piperitenone oxides. Similar behaviour was also detected in accession B, in which piperitone oxide was the major compound together with pulegone and piperitenone oxide. As for accession $\mathrm{C}$, carvone acetate showed a considerable increase (6.0 to 20.1\%) during the vegetative growing stage, while the amount of the other major compound ( $\alpha$-terpineol acetate) remained stable. Slight or no significant changes were observed in accession D (Z-dihydrocarvone as major compound, $68.3-73.7 \%$ ). Significant changes in the major compounds of accession $\mathrm{E}$ (pulegone and isomenthone) were also observed during the initial vegetative growing period. In general, the most noticeable changes were detected at the beginning of the vegetative cycle, whereas the flowering stage showed more stable EO composition. The knowledge of seasonal changes for selected EO chemical profiles should be emphasized because the biological activities are linked with them.

Keywords: Mentha longifolia L., essential oil, TLC profiles, seasonal variations, piperitenone oxide, 


\section{Introduction}

The chemical composition of essential oils (EOs) is subjected to different sources of variability affecting their applications, mainly, those based on their biological activities and, therefore, their quality and price (Figueiredo, 2008). For this reason, the cultivation of medicinal and aromatic plants (MAPs) is needed to obtain uniform and well-known characterized EOs chemical profiles as key requirement for their standardization (Bernath, 2001). Thus, improved yield and EO quality for a safe, effective and foreseeable use can be achieved. On the other hand, developing cultivars for market demands according to new applications based on EO biological activities usually requires domesticating wild plants. For this purpose, the frequent great differences among single plants in wild populations should be considered (Nemeth, 2015). This intrapopulational variability should not be considered only as an obstacle when obtaining representative samples of a population, but rather as an opportunity to take advantage of natural biodiversity.

As emphasized by Schippmann et al. (2002), this genetic and chemical diversity offers the opportunity of obtaining cultivars with suitable EO profiles related to specific biological activities (Gupta, 2017). Likewise, the characterization and propagation of individuals with specific EO profiles can contribute to the conservation of biodiversity and sustainability of wild MAPs collection (Lange et al., 2002; Canter et al., 2005; Schippmann et al., 2006; Lubbe and Verpoorte, 2011).

As above mentioned, cultivating MAPs requires controlling the multiple sources of variation that affect the final quality of the EOs. This management is based on the results of research on the effects of variability factors. Therefore, for research purposes, the differences between the profiles of individual plants should be even more considered. Thus, for example, the results of researches on the effects of seasonal and environmental factors based on the analysis of bulk samples of chemically heterogeneous accessions can lead to misunderstand the meaning of the results (Nemeth, 2015). Obviously, individual monitoring is a good solution, but it has several drawbacks such as the lack of availability of sufficiently large samples or their high number. Obtaining chemically homogeneous experimental cultivars coming from wild populations through a preliminary analysis can be an appropriate alternative, especially when dealing with species in which vegetative propagation can be carried out. Due to its simplicity, availability and reliability, thin layer chromatography (TLC) can be a useful exploration method (Pothier et al., 2001, Hawryl, 2015) and suitable for field research (Franz, 2010). As reported in a previous study (Llorens-Molina et al., 2017), a screening method combining TLC and gas chromatography (GC/MS) analysis of leaves extracts was applied to identify the different EO profiles coming from a wild population of Mentha longifolia (L.) L. located in Teruel (Spain).

As described in the literature, EO from M. longifolia shows a well-known chemodiversity (Lawrence, 2006; Sharopov et al., 2012; Abedi et al., 2015). Two principal chemical profiles can be distinguished according to the molecular skeleton of their main components: a) structures based on menthane skeleton oxygenated in $\mathrm{C} 2$, as those containing carvone and dihydrocarvone (cis and trans isomers) as major compounds (Kokkini et al., 1995; Mastelic et al., 2002; Dzamic et al., 2010; Bertoli et al., 2011), and b) profiles based in menthane skeleton oxygenated in $\mathrm{C}_{3}$, in which two typical profiles related to alternative metabolic pathways involving the double bond $\mathrm{C}_{4}-\mathrm{C}_{8}$ reduction can be distinguished: piperitenone, piperitone and their epoxides (Maffei, 
1988; Saeidi et al., 2012; Segev et al., 2012; Jamzad et al., 2013; Golparvar. 2013; Moradalizadeh et al., 2014) and pulegone, menthone or isomenthone, menthofuran and other menthol derivatives as well (Mkaddem et al., 2009; Hajlaoui et al., 2009; Segev et al., 2012; Salman, 2015). 1,8-cineole is usually found in relatively high proportions in chemotypes characterized by the aforementioned molecular structures (Koliopoulos et al., 2010; Moradalizadeh et al., 2014) and even as the major component in some cases (Fleisher and Fleisher, 1998). On the other hand, $\alpha$-terpinyl acetate and terpinen-4-ol (menthane skeleton oxygenated in $\mathrm{C}_{4}$ ) are reported by Baser et al. (1999) as major compounds in samples from Turkey. Other molecular structures based on the sabinene skeleton have been also referred (Kokkini and Papageorgiou, 1989) as those characterized by $(E)$ sabinen hydrate (Baser et al., 1999). Samples rich in borneol (bornane skeleton) from Pakistan (Hussain, 2010) or linalool (acyclic structure) in Turkey (Baser et al., 1999) have been also reported. Regarding seasonal changes of $M$. longifolia EO there is very little information available. As reported by Hussain (2010), some significant variations between summer and winter were found in wild populations from Pakistan rich in piperitenone oxide, piperitenone and borneol.

The aim of this research was the analysis of the seasonal changes of $M$. longifolia EO yield and composition of five accessions with different profiles grown in experimental plots subjected to the same soil and climate conditions. Four of them could be related with well-defined chemotypes in the literature. Nevertheless, the one rich in $\alpha$-terpineol acetate and carvone acetate has not been reported so far.

\section{Material and methods}

\section{Plant material}

Five accessions of M. longifolia were grown since 2015 at the experimental field of Universitat Politècnica de València (Valencia, Spain) (39 29’02” N; 0 20’13”' W. 4 m a. s. 1.). As described by Llorens-Molina et al. (2017), individuals with similar composition were grouped after TLC-GC/MS characterization of EO profiles and planted in separated experimental plots, hereinafter named A, B, C, D and E accessions. They were placed in such a way that they had the same soil and sun exposition conditions. Irrigation was applied regularly throughout the whole experimental period (April-July, 2017) according to the plants' requirements. Voucher specimens belonging to each profile were kept at the Herbarium of the Universitat Politècnica de València (VALA. No.....................). Meteorological data were collected from the nearest climatic station $(1 \mathrm{~km}$ from the experimental area) (code 08285).

Three samples of leaves (10-15 g) from each accession were collected on the following dates: 04/05/2017 (beginning of vegetative development), 05/06/2017 (full vegetative development), 06/22/2017 (full flowering stage) and 07/06/2017 (advanced flowering stage). They were immediately kept in sealed polyethylene bags and frozen at $-40^{\circ} \mathrm{C}$ until $\mathrm{EO}$ extraction.

\section{Essential Oil extraction}


The samples were subjected for $3 \mathrm{~h}$ to simultaneous extraction distillation using a Likens-Nickerson type apparatus (Chaintreau, 2001). The extracts were dried with anhydrous sodium sulphate and evaporated under reduced pressure at room temperature. After adding $1.5 \mathrm{~mL}$ of dichloromethane ( $\geq 99.9 \%$, capillary GC grade, Sigma-Aldrich ${ }^{\mathrm{TM}}$ ) the extracts were kept in sealed chromatographic vials and stored $-18^{\circ} \mathrm{C}$ until further analysis. The EO yield was determined based on fresh weight of samples by addition of $0.2 \mu \mathrm{g}$ of heptadecane (Sigma-Aldrich ${ }^{\mathrm{TM}}$ ) as internal standard.

\section{GC and GC/MS Analysis}

The analysis of samples was carried out by gas chromatography with flame ionization detector (GC-FID) and mass spectrometry (GC-MS). A Clarus 500 GC (Perkin-Elmer Inc. Wellesley. PA. USA) chromatograph equipped with a FID detector and capillary column ZB-5 (30 m $\times 0.25 \mathrm{~mm}$ i.d. $\times 0.25 \mu \mathrm{m}$ film thickness; Phenomenex Inc. Torrance. CA. USA) was used for quantitative analysis. The injection volume was $1 \mu \mathrm{L}$. The $\mathrm{GC}$ oven temperature was programmed from $50^{\circ} \mathrm{C}$ to $250^{\circ} \mathrm{C}$ at a rate of $3^{\circ} \mathrm{C}$ min- 1 . Helium was the carrier gas $(1.2 \mathrm{~mL} \mathrm{~min}-1)$. Injector and detector temperatures were set at $250^{\circ} \mathrm{C}$. The percentage composition of the EO was calculated from GC peak areas without correction factors by means of the software Total Chrom 6.2 (Perkin-Elmer Inc., Wellesley. PA. USA).

Analysis by GC-MS was performed using a Clarus 500 GC-MS (Perkin-Elmer Inc.) apparatus equipped with the same capillary column, carrier and operating conditions described above for GC-FID analysis. Ionization source temperature was set at $200^{\circ} \mathrm{C}$ and $70 \mathrm{eV}$ electron impact mode was employed. MS spectra were obtained by means of total ion scan (TIC) mode (mass range $\mathrm{m} / \mathrm{z}$ 45-500 uma). The total ion chromatograms and mass spectra were processed with the Turbomass 5.4 software (Perkin-Elmer Inc.). Retention indices were determined by injection of $\mathrm{C}_{8}-\mathrm{C}_{25}$ n-alkanes standard (Supelco, Bellefonte, PE, USA) under the same conditions. The EO components were identified by comparison of calculated retention indices and high probability matches according to mass spectra computer library search (NIST MS 2.0) and available data from literature (Adams, 2007). Identification of the following compounds was also confirmed by comparison of their experimental lineal retention index (LRI) with those of authentic reference standards (Sigma-Aldrich ${ }^{\mathrm{TM}}$ ): $\alpha$-pinene, $\beta$-pinene, camphene, myrcene, limonene, $(Z)-\beta$-ocimene, camphor, terpinolene and terpinen- 4 -ol.

\section{Statistical processing of data}

One-way ANOVA analysis followed by multiple range tests were applied to determine the significance of differences in yield and composition among the sampling times using Statgraphics Centurion XVI® (Statpoint Technologies. Inc.). Tukey's HSD (honest significant difference) test (at $\mathrm{P}<0.05$ ) was applied to evaluate the significance of the differences. To fulfill the homocedasticity requirement, original percentage data were subjected to arcsin [square root $(\% / 100)]$ transformation.

\section{Results}

Results of yield and composition for the four sampling times from the five accessions are displayed in Table 
1. It includes all compounds that reach at least $2 \%$ of the EO composition at some point of sampling and it shows the values for the compounds grouped in classes. The detailed whole compositions of all samples are reported in supplementary materials. Regarding EO yield, the five profiles showed a similar seasonal variation: the lower yield was found at initial vegetative development. It increased during the vegetative period, holding its value up to advanced flowering stage, in which the maximum yield was found. The comparison among the yields for the five accessions is displayed in Figure 1. As the maximum yield value was always reached at the advanced flowering stage, it has been taken as the reference. Accordingly, accession D provided significantly the highest yield, whereas accession $\mathrm{C}$ was the least productive.

Table 1. Seasonal variations of selected EO profiles of Mentha longifolia (L) L.

\begin{tabular}{|c|c|c|c|c|}
\hline Sampling number & S1 & $\mathrm{S} 2$ & S3 & S4 \\
\hline Sampling date & April/5/2017 & May/30/2017 & June/22/2017 & July/6/2017 \\
\hline Phenological stage & $\begin{array}{l}\text { Initial } \\
\text { vegetative } \\
\text { development }\end{array}$ & \begin{tabular}{|l|} 
Full \\
vegetative \\
development \\
\end{tabular} & \begin{tabular}{|l|} 
Full \\
flowering \\
beginning \\
\end{tabular} & $\begin{array}{l}\text { Advanced ful } \\
\text { flowering }\end{array}$ \\
\hline EO PROFILE A & \multicolumn{4}{|c|}{ COMPOSITION (yield as $m \mathrm{EO} / m$ (fresh leaves)) } \\
\hline Yield & $0.4 \pm 0.2 \mathrm{a}$ & $0.6 \pm 0.2 \mathrm{ab}$ & $0.6 \pm 0.1 \mathrm{ab}$ & $0.8 \pm 0.1 \mathrm{~b}$ \\
\hline$\alpha$-Pinene & $1.9 \pm 0.1 \mathrm{a}$ & $1.0 \pm 0.1 \mathrm{~b}$ & $0.8 \pm 0.2 \mathrm{~b}$ & $0.8 \pm 0.1 \mathrm{~b}$ \\
\hline Sabinene & $1.7 \pm 1.1 \mathrm{a}$ & $0.6 \pm 0.1 \mathrm{a}$ & $0.7 \pm 0.1 \mathrm{a}$ & $0.7 \pm 0.1 \mathrm{a}$ \\
\hline$\beta$-Pinene & $1.1 \pm 0.4 \mathrm{a}$ & $1.0 \pm 0.2 \mathrm{a}$ & $1.1 \pm 0.2 \mathrm{a}$ & $1.2 \pm 0.1 \mathrm{a}$ \\
\hline Myrcene & $1.1 \pm 0.3 \mathrm{a}$ & $1.1 \pm 0.2 \mathrm{a}$ & $1.0 \pm 0.2 \mathrm{a}$ & $1.0 \pm 0.1 \mathrm{a}$ \\
\hline Limonene & $3.0 \pm 0.9 \mathrm{a}$ & $2.7 \pm 0.6 \mathrm{a}$ & $1.2 \pm 0.2 \mathrm{~b}$ & $0.9 \pm 0.1 \mathrm{~b}$ \\
\hline$(Z)-\beta$-Ocimene & $2.5 \pm 0.8 \mathrm{a}$ & $2.0 \pm 0.4 \mathrm{a}$ & $1.8 \pm 0.4 \mathrm{a}$ & $1.6 \pm 0.0 \mathrm{a}$ \\
\hline Hydrocarbon monoterpenes & $11.6 \pm 3.6 \mathrm{a}$ & $8.8 \pm 1.7 \mathrm{ab}$ & $6.9 \pm 1.5 \mathrm{ab}$ & $6.4 \pm 0.3 \mathrm{~b}$ \\
\hline 1,8-Cineole & $5.8 \pm 3.6 \mathrm{a}$ & $4.2 \pm 1.4 \mathrm{a}$ & $4.1 \pm 0.7 \mathrm{a}$ & $5.0 \pm 0.8 \mathrm{a}$ \\
\hline Piperitone oxide & $63.3 \pm 4.1 \mathrm{a}$ & $31.5 \pm 5.7 \mathrm{~b}$ & $22.6 \pm 1.6 \mathrm{c}$ & $27.0 \pm 1.9 \mathrm{bc}$ \\
\hline Piperitenone oxide & $7.1 \pm 1.2 \mathrm{a}$ & $42.0 \pm 5.7 \mathrm{~b}$ & $55.7 \pm 4.4 \mathrm{c}$ & $49.8 \pm 2.7 \mathrm{c}$ \\
\hline Oxigenated monoterpenes & $77.5 \pm 0.1 \mathrm{a}$ & $79.5 \pm 2.9 \mathrm{a}$ & $83.6 \pm 2.0 \mathrm{~b}$ & $83.1 \pm 0.8 \mathrm{~b}$ \\
\hline$\beta$-Caryophyllene & $2.6 \pm 0.4 \mathrm{a}$ & $3.6 \pm 0.7 \mathrm{~b}$ & $3.1 \pm 0.2 \mathrm{ab}$ & $3.4 \pm 0.6 \mathrm{ab}$ \\
\hline Germacrene-D & $4.4 \pm 2.4 \mathrm{a}$ & $5.4 \pm 0.6 \mathrm{a}$ & $4.0 \pm 0.4 \mathrm{a}$ & $3.8 \pm 0.1 \mathrm{a}$ \\
\hline Hydrocarbon sesquiterpenes & $8.2 \pm 3.6 \mathrm{a}$ & $10.0 \pm 1.0 \mathrm{a}$ & $8.1 \pm 0.8 \mathrm{a}$ & $8.4 \pm 0.6 \mathrm{a}$ \\
\hline Oxigenated sesquiterpenes & $0.5 \pm 0.4 \mathrm{a}$ & $0.4 \pm 0.1 \mathrm{a}$ & $0.3 \pm 0.0$ a & $0.3 \pm 0.1 \mathrm{a}$ \\
\hline Other compounds & $1.2 \pm 0.6 \mathrm{a}$ & $0.7 \pm 0.3 \mathrm{a}$ & $0.7 \pm 0.2 \mathrm{a}$ & $1.0 \pm 0.0 \mathrm{a}$ \\
\hline Total identified & $99.0 \pm 0.3 \mathrm{a}$ & $99.4 \pm 0.3 \mathrm{a}$ & $99.5 \pm 0.1 \mathrm{a}$ & $99.3 \pm 0.1 \mathrm{a}$ \\
\hline
\end{tabular}

Table 1 (cont.)

\begin{tabular}{|l|l|l|l|l|}
\hline \multicolumn{1}{|l|}{ EO PROFILE B } & $\mathbf{0 . 4} \pm \mathbf{0 . 1} \mathbf{a}$ & $\mathbf{0 . 8} \pm \mathbf{0 . 1}$ bc & $\mathbf{0 . 6} \pm \mathbf{0 . 1} \mathbf{a b}$ & $\mathbf{0 . 9} \pm \mathbf{0 . 2} \mathbf{~ c}$ \\
\hline yield & $2.1 \pm 0.4 \mathrm{a}$ & $0.7 \pm 0.1 \mathrm{~b}$ & $0.5 \pm 0.1 \mathrm{~b}$ & $0.6 \pm 0.0 \mathrm{~b}$ \\
\hline$\alpha$-Pinene & $0.4 \pm 0.1 \mathrm{a}$ & $0.4 \pm 0.1 \mathrm{a}$ & $0.5 \pm 0.0 \mathrm{a}$ & $0.5 \pm 0.0 \mathrm{a}$ \\
\hline Sabinene & $0.7 \pm 0.1 \mathrm{ab}$ & $0.6 \pm 0.1 \mathrm{a}$ & $0.8 \pm 0.0 \mathrm{ab}$ & $0.8 \pm 0.1 \mathrm{~b}$ \\
\hline$\beta$-Pinene & $0.8 \pm 0.1 \mathrm{a}$ & $0.6 \pm 0.1 \mathrm{~b}$ & $0.7 \pm 0.0 \mathrm{ab}$ & $0.7 \pm 0.1 \mathrm{ab}$ \\
\hline Myrcene & $4.1 \pm 1.3 \mathrm{a}$ & $3.6 \pm 0.5 \mathrm{a}$ & $3.8 \pm 0.4 \mathrm{a}$ & $3.6 \pm 0.4 \mathrm{a}$ \\
\hline Limonene & - & - & - & $t$ \\
\hline (Z)- $\beta$-Ocimene & & & & \\
\hline
\end{tabular}




\begin{tabular}{|c|c|c|c|c|}
\hline Hydrocarbon monoterpenes & $8.3 \pm 1.3 \mathrm{a}$ & $6.0 \pm 0.8 \mathrm{~b}$ & $6.4 \pm 0.4 \mathrm{~b}$ & $6.3 \pm 0.7 \mathrm{~b}$ \\
\hline 1,8-Cineole & $1.7 \pm 0.3 \mathrm{a}$ & $1.0 \pm 0.1 \mathrm{~b}$ & $1.0 \pm 0.1 \mathrm{~b}$ & $1 . \pm 0.1 \mathrm{~b}$ \\
\hline Pulegone & $10.1 \pm 1.8 \mathrm{a}$ & $\begin{array}{ll}16.3 \pm 14.9 \\
a\end{array}$ & $23.8 \pm 6.0 \mathrm{a}$ & $17.8 \pm 4.1 \mathrm{a}$ \\
\hline Piperitone oxide & $61.0 \pm 1.8 \mathrm{a}$ & $\begin{array}{l}42.0 \pm 14.2 \\
b\end{array}$ & $\begin{array}{l}46.6 \pm 2.6 \\
\mathrm{ab}\end{array}$ & $52.8 \pm 3.5 \mathrm{ab}$ \\
\hline Piperitenone oxide & $2.8 \pm 1.7 \mathrm{a}$ & $18.3 \pm 5.2 \mathrm{~b}$ & $8.1 \pm 5.3 \mathrm{a}$ & $3.5 \pm 1.5 \mathrm{a}$ \\
\hline Oxygenated monoterpenes & $78.4 \pm 1.3 \mathrm{a}$ & $\begin{array}{l}80.6 \pm 1.0 \\
\text { ab }\end{array}$ & $81.5 \pm 1.5 b$ & $81.1 \pm 1.5 b$ \\
\hline$\beta$-Caryophyllene & $2.4 \pm 1.1 \mathrm{a}$ & $2.8 \pm 0.2 \mathrm{a}$ & $2.5 \pm 0.4 \mathrm{a}$ & $1.8 \pm 1.3 \mathrm{a}$ \\
\hline Germacrene-D & $6.0 \pm 1.4 \mathrm{a}$ & $7.1 \pm 0.6 \mathrm{a}$ & $6.5 \pm 1.1 \mathrm{a}$ & $5.7 \pm 0.4 \mathrm{a}$ \\
\hline Hydrocarbon sesquiterpenes & $9.6 \pm 2.8 \mathrm{a}$ & $10.7 \pm 0.8 \mathrm{a}$ & $9.8 \pm 1.7 \mathrm{a}$ & $9.3 \pm 1.9 \mathrm{a}$ \\
\hline Oxygenated sesquiterpenes & $1.0 \pm 0.1 \mathrm{a}$ & $0.9 \pm 0.2 \mathrm{a}$ & $0.8 \pm 0.2 \mathrm{a}$ & $0.8 \pm 0.2 \mathrm{a}$ \\
\hline Other compounds & $1.2 \pm 0.1 \mathrm{a}$ & $0.8 \pm 0.1 \mathrm{~b}$ & $0.7 \pm 0.2 \mathrm{~b}$ & $1.4 \pm 0.1 \mathrm{a}$ \\
\hline Total identified & 98.5 & 99.0 & 99.3 & 99.0 \\
\hline
\end{tabular}

Table 1 (cont.)

\begin{tabular}{|c|c|c|c|c|}
\hline \multicolumn{5}{|l|}{ EO PROFILE C } \\
\hline Yield & $0.1 \pm 0.0 \mathrm{a}$ & $0.2 \pm 0.0 \mathrm{~b}$ & $0.2 \pm 0.1 \mathrm{~b}$ & $0.3 \pm 0.0 \mathrm{~b}$ \\
\hline$\alpha$-Pinene & $3.8 \pm 0.6 \mathrm{a}$ & $0.8 \pm 0.1 \mathrm{~b}$ & $0.2 \pm 0.0 \mathrm{c}$ & $0 . \pm 0.0 \mathrm{c}$ \\
\hline Sabinene & $4.4 \pm 0.8 \mathrm{a}$ & $2.7 \pm 0.7 b$ & $3.0 \pm 0.7 \mathrm{ab}$ & $3.5 \pm 0.5 \mathrm{ab}$ \\
\hline$\beta$-Pinene & $0.6 \pm 0.1 \mathrm{a}$ & $0.4 \pm 0.1 \mathrm{~b}$ & $0.4 \pm 0.1 \mathrm{ab}$ & $0.5 \pm 0.1 \mathrm{ab}$ \\
\hline Myrcene & $2.6 \pm 0.4 \mathrm{a}$ & $1.8 \pm 0.4 \mathrm{~b}$ & $1.7 \pm 0.4 \mathrm{~b}$ & $1.8 \pm 0.2 \mathrm{~b}$ \\
\hline Limonene & $1.6 \pm 0.4 \mathrm{a}$ & $0.5 \pm 0.1 \mathrm{~b}$ & $0.7 \pm 0.3 \mathrm{~b}$ & $0.6 \pm 0.1 \mathrm{~b}$ \\
\hline (Z)- $\beta$-Ocimene & $1.3 \pm 0.2 \mathrm{a}$ & $1.0 \pm 0.2 \mathrm{ab}$ & $0.8 \pm 0.2 \mathrm{bc}$ & $0.6 \pm 0.1 \mathrm{c}$ \\
\hline Hydrocarbon monoterpenes & $15.3 \pm 2.3 \mathrm{a}$ & $7.6 \pm 1.5 \mathrm{~b}$ & $7.4 \pm 1.9 \mathrm{~b}$ & $7.6 \pm 1.2 \mathrm{~b}$ \\
\hline 1,8-Cineole & $6.4 \pm 0.7 \mathrm{a}$ & $4.4 \pm 0.7 \mathrm{a}$ & $5.4 \pm 1.6 \mathrm{a}$ & $5.0 \pm 0.9 \mathrm{a}$ \\
\hline$\alpha$-Terpineol & $3.6 \pm 0.7 \mathrm{a}$ & $6.8 \pm 1.2 \mathrm{~b}$ & $2.6 \pm 0.6 \mathrm{a}$ & $0.8 \pm 0.2 \mathrm{c}$ \\
\hline Carvone & $1.5 \pm 1.3 \mathrm{a}$ & $3.5 \pm 0.9 \mathrm{~b}$ & $3.4 \pm 0.9 \mathrm{~b}$ & $4.0 \pm 0.4 \mathrm{~b}$ \\
\hline Piperitone oxide & $4.1 \pm 4.6 \mathrm{a}$ & $0.2 \pm 0.2 \mathrm{~b}$ & $0.0 \pm 0.0 \mathrm{~b}$ & $0.8 \pm 0.8 \mathrm{~b}$ \\
\hline$\alpha$-Terpineol acetate & $48.3 \pm 5.6 \mathrm{a}$ & $42.1 \pm 6.8 \mathrm{a}$ & $48.8 \pm 8.8 \mathrm{a}$ & $47.7 \pm 2.4 \mathrm{a}$ \\
\hline Carvone acetate & $6.0 \pm 1.9 \mathrm{a}$ & $20.1 \pm 9.1 \mathrm{~b}$ & $\begin{array}{l}17.4 \pm 14.1 \\
\mathrm{ab}\end{array}$ & $17 . \pm 3.6 \mathrm{ab}$ \\
\hline Oxygenated monoterpenes & $77.1 \pm 3.8 \mathrm{a}$ & $83.0 \pm 2.1 \mathrm{ab}$ & $84.2 \pm 2.9 \mathrm{~b}$ & $82.3 \pm 1.6 \mathrm{~b}$ \\
\hline$\beta$-Caryophyllene & $1.2 \pm 0.3 \mathrm{a}$ & $2.7 \pm 0.5 \mathrm{a}$ & $3.1 \pm 0.4 \mathrm{a}$ & $3.3 \pm 0.1 \mathrm{a}$ \\
\hline Germacrene-D & $1.6 \pm 1.0 \mathrm{a}$ & $2.1 \pm 0.3 \mathrm{a}$ & $1.3 \pm 0.1 \mathrm{a}$ & $1.3 \pm 0.1 \mathrm{a}$ \\
\hline Hydrocarbon sesquiterpenes & $3.6 \pm 1.4 \mathrm{a}$ & $6.6 \pm 1.1 \mathrm{~b}$ & $6.9 \pm 0.8 \mathrm{~b}$ & $6.2 \pm 1.9 \mathrm{~b}$ \\
\hline Oxygenated sesquiterpenes & $0.2 \pm 0.1 \mathrm{a}$ & $0.2 \pm 0.1 \mathrm{a}$ & $0.1 \pm 0.0 \mathrm{a}$ & $0.3 \pm 0.1 \mathrm{a}$ \\
\hline Other compounds & $2.1 \pm 0.2 \mathrm{a}$ & $1.4 \pm 0.2 \mathrm{ab}$ & $0.7 \pm 0.4 \mathrm{~b}$ & $2.6 \pm 1.8 \mathrm{a}$ \\
\hline Total identified & 98.4 & 98.8 & 99.2 & 99.0 \\
\hline
\end{tabular}

\section{EO PROFILE D}

\begin{tabular}{|l|l|l|l|l|}
\hline Yield & $\mathbf{0 . 7} \pm \mathbf{0 . 1} \mathbf{a}$ & $\mathbf{0 . 9} \pm \mathbf{0 . 1} \mathbf{a}$ & $\mathbf{0 . 8} \pm \mathbf{0 . 0} \mathbf{a}$ & $\mathbf{1 . 2} \pm \mathbf{0 . 1} \mathbf{b}$ \\
\hline$\alpha$-Pinene & $1.2 \pm 0.8 \mathrm{a}$ & $0.8 \pm 0.1 \mathrm{a}$ & $0.7 \pm 0.1 \mathrm{a}$ & $0.7 \pm 0.1 \mathrm{a}$ \\
\hline Sabinene & $0.8 \pm 0.1 \mathrm{a}$ & $0.7 \pm 0.1 \mathrm{a}$ & $0.8 \pm 0.1 \mathrm{a}$ & $0.8 \pm 0.1 \mathrm{a}$ \\
\hline$\beta$-Pinene & $0.9 \pm 0.0 \mathrm{a}$ & $0.9 \pm 0.1 \mathrm{a}$ & $1.0 \pm 0.1 \mathrm{a}$ & $1.0 \pm 0.1 \mathrm{a}$ \\
\hline Myrcene & $0.8 \pm 0.1 \mathrm{a}$ & $0.9 \pm 0.1 \mathrm{a}$ & $0.9 \pm 0.1 \mathrm{a}$ & $0.9 \pm 0.1 \mathrm{a}$ \\
\hline
\end{tabular}




\begin{tabular}{|l|l|l|l|l|}
\hline Limonene & $6.1 \pm 1.1 \mathrm{a}$ & $6.5 \pm 0.4 \mathrm{a}$ & $6.7 \pm 1.0 \mathrm{a}$ & $5.0 \pm 1.0 \mathrm{a}$ \\
\hline$(\mathrm{Z})-\beta$-Ocimene & $1.4 \pm 0.0 \mathrm{a}$ & $1.0 \pm 0.1 \mathrm{~b}$ & $0.8 \pm 0.1 \mathrm{c}$ & $0.8 \pm 0.1 \mathrm{c}$ \\
\hline Hydrocarbon monoterpenes & $\mathbf{6 . 1} \pm \mathbf{1 . 1} \mathbf{~ a}$ & $\mathbf{6 . 5} \pm \mathbf{0 . 4} \mathbf{~ a b}$ & $\mathbf{6 . 7} \pm \mathbf{1 . 0} \mathbf{a b}$ & $\mathbf{5 . 0} \pm \mathbf{1 . ~ b}$ \\
\hline 1,8 -Cineole & $5.7 \pm 0.5 \mathrm{a}$ & $5.6 \pm 0.2 \mathrm{a}$ & $5.9 \pm 0.4 \mathrm{a}$ & $5.4 \pm 0.4 \mathrm{a}$ \\
\hline Z)-Dihydrocarvone & $68.3 \pm 2.5 \mathrm{a}$ & $71.7 \pm 1.2 \mathrm{~b}$ & $73.7 \pm 1.4 \mathrm{~b}$ & $73.3 \pm 1.4 \mathrm{~b}$ \\
\hline Oxygenated monoterpenes & $\mathbf{7 8 . 4} \pm \mathbf{2 . 6} \mathbf{a}$ & $\mathbf{7 9 . 8} \pm \mathbf{1 . 0} \mathbf{a b}$ & $\mathbf{8 2 . 1} \pm \mathbf{1 . 7} \mathbf{b}$ & $\mathbf{8 2 . 5} \pm \mathbf{0 . 6} \mathbf{b}$ \\
\hline$\beta$-Caryophyllene & $2.4 \pm 0.6 \mathrm{a}$ & $2.5 \pm 0.5 \mathrm{a}$ & $2.1 \pm 0.4 \mathrm{a}$ & $2.5 \pm 0.2 \mathrm{a}$ \\
\hline Germacrene-D & $5.6 \pm 1.5 \mathrm{a}$ & $4.9 \pm 1.0 \mathrm{ab}$ & $3.0 \pm 1.2 \mathrm{~b}$ & $3.5 \pm 0.4 \mathrm{ab}$ \\
\hline Hydrocarbon sesquiterpenes & $\mathbf{8 . 0} \pm \mathbf{2 . 1} \mathbf{a}$ & $\mathbf{7 . 4} \pm \mathbf{1 . 5} \mathbf{a b}$ & $\mathbf{5 . 1} \pm \mathbf{1 . 5} \mathbf{b}$ & $\mathbf{5 . 9} \pm \mathbf{0 . 6} \mathbf{~ a b}$ \\
\hline Oxygenated sesquiterpenes & $\mathbf{0 . 2} \pm \mathbf{0 . 1} \mathbf{a}$ & $\mathbf{0 . 2} \pm \mathbf{0 . 0} \mathbf{a}$ & $\mathbf{0 . 3} \pm \mathbf{0 . 2} \mathbf{a}$ & $\mathbf{0 . 4} \pm \mathbf{0 . 1} \mathbf{~ a}$ \\
\hline Other compounds & $\mathbf{0 . 8} \pm \mathbf{0 . 1} \mathbf{a b}$ & $\mathbf{0 . 9} \pm \mathbf{0 . 1} \mathbf{a b}$ & $\mathbf{0 . 7} \pm \mathbf{0 . 0} \mathbf{a}$ & $\mathbf{0 . 9} \pm \mathbf{0 . 2} \mathbf{b}$ \\
\hline Total identified & $99.6 \pm 0.2$ & $99.8 \pm 0.0$ & $99.7 \pm 0.1$ & $99.4 \pm 0.5$ \\
\hline
\end{tabular}

Table 1 (con.)

\begin{tabular}{|c|c|c|c|c|}
\hline \multicolumn{5}{|l|}{ EO Profile E } \\
\hline Yield & $0.5 \pm 0.1$ a & $0.7 \pm 0.0 \mathrm{~b}$ & $0.7 \pm 0.1 \mathrm{~b}$ & $0.8 \pm 0.1 \mathrm{~b}$ \\
\hline$\alpha$-Pinene & $0.6 \pm 0.1 \mathrm{ab}$ & $0.5 \pm 0.1 \mathrm{a}$ & $0.7 \pm 0.1 \mathrm{~b}$ & $0.5 \pm 0.1 \mathrm{a}$ \\
\hline Sabinene & $0.6 \pm 0.0 \mathrm{a}$ & $0.4 \pm 0.1 \mathrm{~b}$ & $0.5 \pm 0.0 \mathrm{a}$ & $0.4 \pm 0.0 \mathrm{~b}$ \\
\hline$\beta$-Pinene & $0.6 \pm 0.1 \mathrm{a}$ & $0.6 \pm 0.1 \mathrm{a}$ & $0.9 \pm 0.1 \mathrm{~b}$ & $0.7 \pm 0.1 \mathrm{a}$ \\
\hline Myrcene & $0.6 \pm 0.1 \mathrm{a}$ & $0.6 \pm 0.1 \mathrm{a}$ & $0.8 \pm 0.1 \mathrm{~b}$ & $0.6 \pm 0.0 \mathrm{a}$ \\
\hline Limonene & $1.6 \pm 0.4 \quad \mathrm{a}$ & $1.4 \pm 0.0 \mathrm{a}$ & $0.9 \pm 0.2 \mathrm{~b}$ & $0.7 \pm 0.1 \mathrm{~b}$ \\
\hline (Z)- $\beta$-ocimene & $2.1 \pm 0.1 \mathrm{a}$ & $1.6 \pm 0.3 \mathrm{a}$ & $1.6 \pm 0.0 \mathrm{a}$ & $0.8 \pm 0.4 \mathrm{~b}$ \\
\hline Hydrocarbon monoterpenes & $7.0 \pm 0.7 \mathrm{a}$ & $5.8 \pm 0.8 \mathrm{a}$ & $6.3 \pm 0.3 \mathrm{a}$ & $4.6 \pm 0.4 \mathrm{~b}$ \\
\hline 1,8-Cineole & $3.5 \pm 0.1 \mathrm{a}$ & $3.0 \pm 0.6 \mathrm{ab}$ & $4.8 \pm 0.3 \mathrm{c}$ & $2.2 \pm 0.8 \mathrm{~b}$ \\
\hline Isomenthone & $27.8 \pm 1.9 \mathrm{a}$ & $\mathrm{t} \quad \mathrm{b}$ & $0.1 \pm 0.0 \mathrm{~b}$ & $11.6 \pm 0.3 \mathrm{c}$ \\
\hline Pulegone & $49.4 \pm 1.1 \mathrm{a}$ & $77.2 \pm 1.0 \mathrm{~b}$ & $73.4 \pm 3.3 \mathrm{bc}$ & $70.3 \pm 2.4 \mathrm{c}$ \\
\hline Oxygenated monoterpenes & $80.7 \pm 0.8 \mathrm{ab}$ & $80.3 \pm 0.4 a$ & $78.2 \pm 3.5 \mathrm{a}$ & $84.1 \pm 2.1 b$ \\
\hline$\beta$-Caryophyllene & $1.7 \pm 0.1 \mathrm{a}$ & $2.6 \pm 0.1 \mathrm{bc}$ & $3.2 \pm 0.8 \mathrm{~b}$ & $2.4 \pm 0.3 \mathrm{c}$ \\
\hline Germacrene-D & $2.9 \pm 0.4 \mathrm{ab}$ & $3.9 \pm 0.3 \mathrm{~b}$ & $2.0 \pm 1.3 \mathrm{a}$ & $2.3 \pm 0.0 \mathrm{a}$ \\
\hline Hydrocarbon sesquiterpenes & $5.1 \pm 0.6 \mathrm{a}$ & $7.2 \pm 0.1 \mathrm{a}$ & $6.2 \pm 2.2 \mathrm{a}$ & $5.4 \pm 0.5 \mathrm{a}$ \\
\hline Oxygenated sesquiterpenes & $0.4 \pm 0.0$ a & $0.4 \pm 0.0$ a & $1.0 \pm 0.1 \mathrm{~b}$ & $0.4 \pm 0.2 \mathrm{a}$ \\
\hline Other compounds & $0.7 \pm 0.0$ a & $0.7 \pm 0.0$ a & $0.7 \pm 0.1$ a & $0.5 \pm 0.1 \mathrm{a}$ \\
\hline Total identified & 99.6 & 99.6 & 99.3 & 99.5 \\
\hline
\end{tabular}

Total yield and relative amounts of grouped and individual compounds (as \% of the total chromatogram). Values in the same line labelled with different letters differed significantly (ANOVA, HSD test at $\mathrm{P}<0.05$ ). 


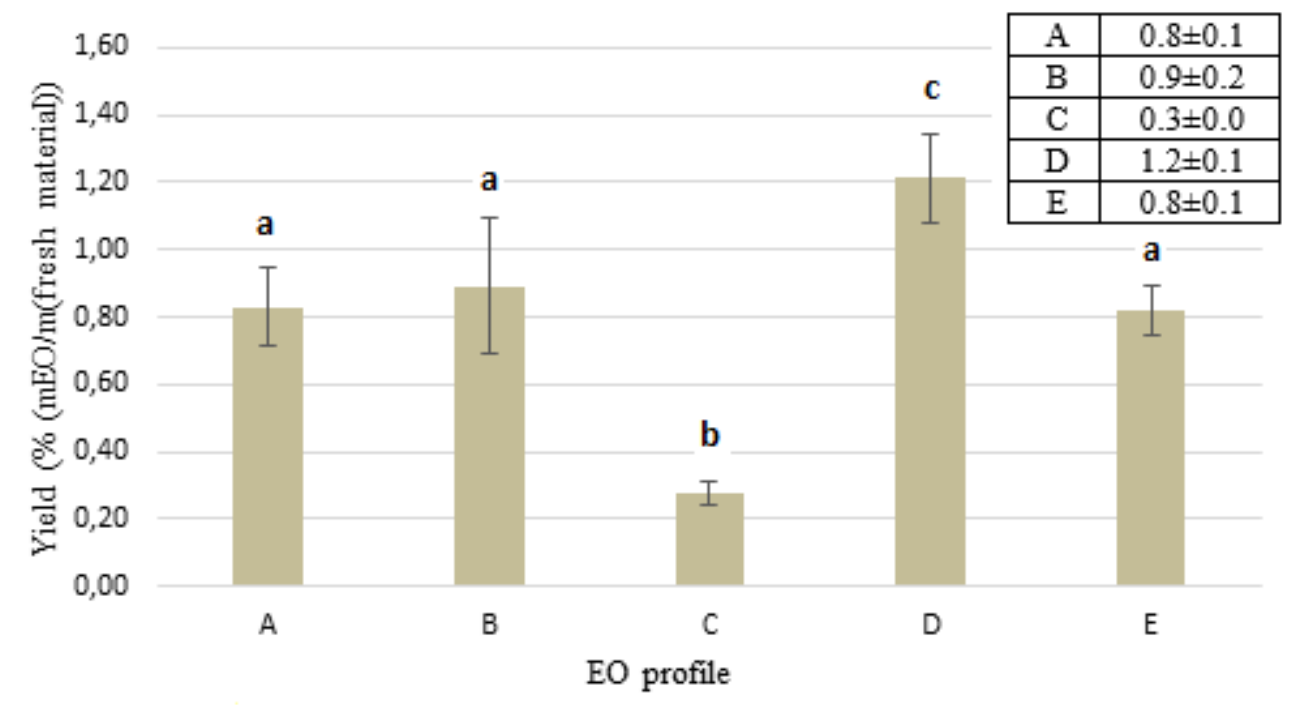

Figure 1 Yield (mean $\pm \mathrm{SD}$ ) values for each accession at the advanced flowering stage (maximum yield). Bars labelled with different letters differ significantly (ANOVA, HSD test at $\mathrm{P}<0.05$ )

Some meteorological data: daily maximum and minimum temperature, daily relative humidity and solar radiation (rainfall was not considered as the experimental plots were irrigated) were taken from the nearest climatic station (Figure 2). Data reflects the progressive increase in temperatures until the full flowering stage. Thereafter, temperatures reach the usual summer stability, although it is interrupted on certain days by the sudden temperature increase that takes place when the wind turns to the west. This phenomenon is usually linked to remarkable drop in relative humidity. Solar radiation changes in a similar way, according to sunlight duration, with isolated cloudy days. In summary, no anomalous long periods able to affect the plant development were registered.

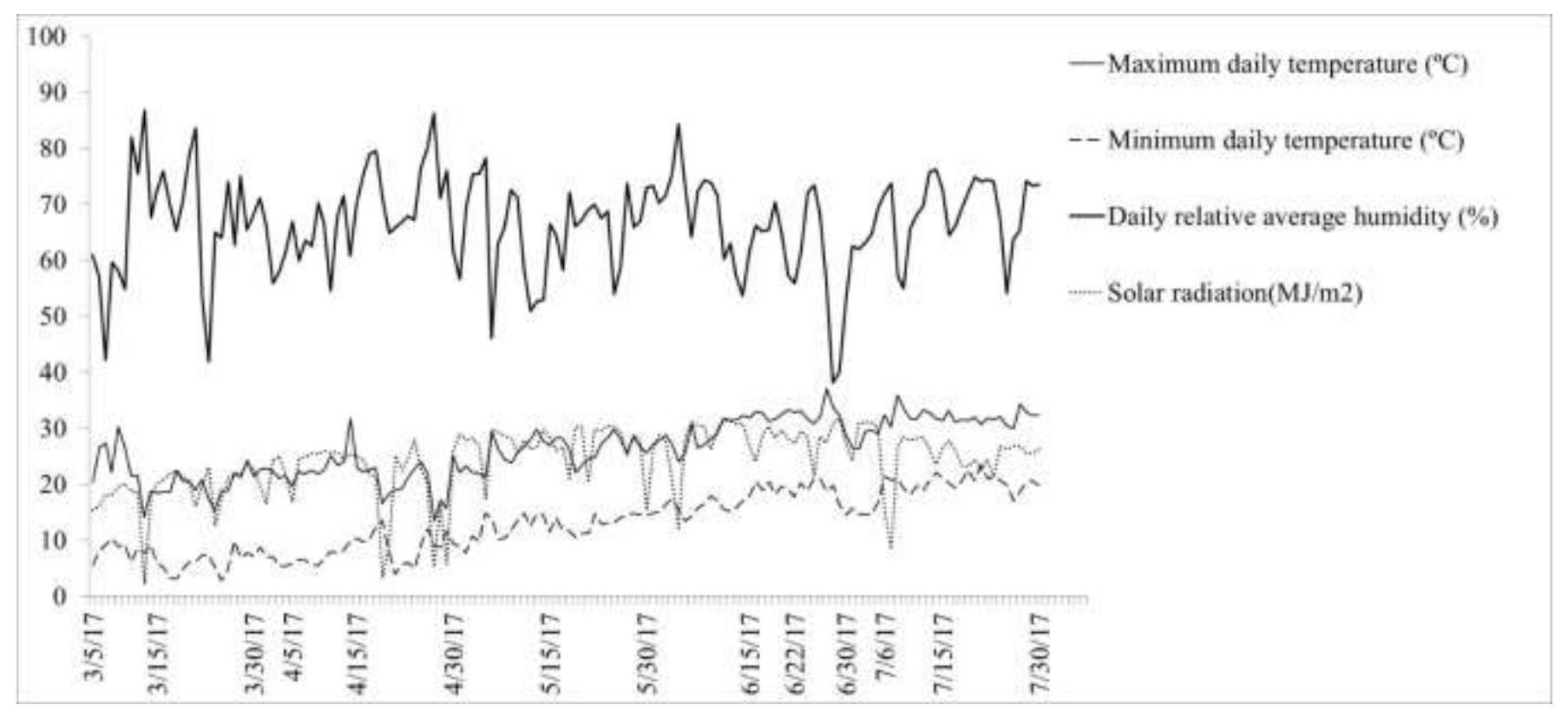

Fig. 2. Meteorological data over the experimental period 


\section{Discussion}

Except for the defined profile $\mathrm{C}$ ( $\alpha$-terpineol acetate + carvone acetate) which has not been described so far in the literature, the rest of profiles could be related with those reported in previous researches. For example, EOs rich in piperitone oxide and piperitenone oxide (profile A) have been reported by some researchers (Sharopov, 2012), but no information about phenological stage in sampling time was available. Only Hussain et al. (2010) distinguished between summer and winter seasons,but considering the geographical origin and the provided meteorological data, the comparison of their results with those obtained in this work is not possible.

Profile B was characterized by piperitone oxide as major compound with appreciable proportions of pulegone and piperitenone oxide. Similar profiles were reported by Gulluce et al., (2007) and Abedi et al. (2015), although in the last one, two of the sampling locations showed high amounts of 1,8-cineole (up to $28.84 \%$ ).

Profile D shows a large proportion of dihydrocarvone (68.3-73.7\%), matching with profiles rich in dihydrocarvone isomers previously reported by Dzamic et al. (2010) and Matovic (1999) in Serbia. Pulegone was found the major compound in profile $\mathrm{E}$, although isomenthone reaches an important amount at the beginning of vegetative cycle. Profiles rich in pulegone have been previously reported by Fleisher and Fleisher (1998), Abedi et al. (2015) in two of the sampling locations and Salman (2015) in samples coming from Saudi Arabia.

Yield values showed noticeable differences among the EO profiles (Figure 1) and also according to sampling time within each accession (0.1-1.2 \% (w EO/ w fresh material) in Table 1). Profile $\mathrm{C}$ had the poorest yield $(0.1-0.3 \%)$ whereas that from profile D gradually increased from $0.7 \%$ at the beginning to $1.2 \%$ at the advanced full flowering. .As displayed in table 1, the five profiles showed a similar seasonal variation: the lower yield was found at initial vegetative development. It increased during the vegetative period, maintaining its value up to advanced flowering stage, in which the maximum yield was found. It is worth to mention the morphologic differences in plants from accession C (lower yield), which were characterized by a higher size and turgidity of leaves, the same way that a greater vegetative development.

As for seasonal composition variations, some regularities were found concerning the grouped compounds. A significant decrease was found in the hydrocarbon monoterpene fraction between the beginning and the end of experimental period for the five profiles. Nevertheless, in A, B, C and D, this decrease was observed at the beginning of the vegetative period, maintaining its proportion relatively stable up to the end of flowering. Although showing a similar evolution, the significant decrease was observed in profile E during the last stage of flowering.

Conversely, the oxygenated monoterpene fraction, which was the major one for the five profiles, increased its proportion over the experimental period, although their significant lower values were found always at the first sampling. The hydrocarbon sesquiterpene fraction could be characterized by its seasonal stability, except for minor although significant changes observed in profiles $\mathrm{C}$ (increasing at the first stage of vegetative 
development) and D (decreasing between the first and third sampling (full flowering)). The rest of fractions showed negligible and practically constant amounts.

The most relevant changes were found in the major compounds, mainly in those belonging to oxygenated monoterpene fraction. Nevertheless, because of the diversity of hydrocarbon monoterpenic composition, all those were found in appreciable amounts at least in one of the profiles as displayed in Table 1.

The compound $\alpha$-pinene showed a significant decrease over the vegetative development in profiles A, B and C. An analogous but not significant change was appreciated in D. A similar pattern of variation was found in $\mathrm{A}$ and $\mathrm{C}$ profiles for sabinene, whereas it was constant in B and D. In the five profiles $\beta$-pinene was found practically unvarying. All these monoterpenes remained stable over the whole experimental period in profile E. More relevant could be considered the variations in limonene amounts, as they were rather higher (up to 6.7 $\%$ in profile D). Except for B and D profiles, in which its proportion remained without statistically significant changes, in the rest of profiles its higher value was noted in the first sampling, reaching its lower value at the end of flowering stage for the profiles A and E. Lastly, a very similar behaviour was observed for (Z)- $\beta$ ocimene, except for profile B in which only traces were found at the end of this stage.

Piperitone oxide and piperitenone oxide changed in opposite way in profile A. The first one decreased from the beginning up to full flowering stage and the second one increased, with a slight and no significant change of tendency when full flowering stage advanced. Changes in piperitone oxide were found analogous in profile $\mathrm{B}$, mainly as regards the initial decrease. Pulegone remained without significant changes over the whole experimental period and piperitenone oxide reached its maximum value at full vegetative stage and decreased at the end of flowering to values similar to those found at the beginning.

Concerning profile $\mathrm{C}, \alpha$-terpineol acetate did not change significantly. The other major compound (carvone acetate) showed a great and significant increase over the vegetative development stage, remaining steady up to the end of flowering stage.

Dihydrocarvone, in profile D, was the predominant compound (68.3-73.7\%) and showed a slight but significant increase when the flowering stage was reached.

As for accession E, the pulegone proportion increased in the vegetative development stages, as it happened, but no significantly, in profile B. A minor but significant decrease was observed from the second to the fourth sampling. It is worth highlighting the changes of isomenthone: $27.8 \%$ at the first sampling, then virtually disappeared up to the end of flowering when reached $11.6 \%$.

Results support the importance of considering the phenological stage when defining chemotypes to obtain standardized EO compositions. Some significant and important changes take place at the beginning of plant development. Likewise, in general, from the full flowering stage on, the composition showed a certain tendency to stabilize. Nevertheless, the occurrence of high levels of certain compounds at the beginning of the 
vegetative cycle should be taken into account owing to their potential usefulness related to their biological activity.

The five studied accessions, even sharing the specific characteristics of $M$. longifolia, showed certain distinctive features in terms of the rhythm and degree of vegetative development. These differences were associated with the significant differences observed in EO yields. These variations should be analyzed considering that the five cultivars were developed under the same conditions of soil, climate and irrigation. This advises to deepen in its morphological study in case there were relevant differences from the taxonomic point of view.

\section{References}

- Abedi, R.,, Golparvar, A.R., Hadipanah, A. 2015. "Identification of the essential oils composition from four ecotypes of Mentha longifolia (L.) Huds. growing wild in Isfahan province, Iran.Journal of BioScience and Biotechnology 4 (2), 117-121.

- Adams, RP. 2007. Identification of essential oil components by gas chromatography/mass spectrometry. Allured Publishing Corporation, Illinois, USA.

- Baser, K.H.C., Kürkçüoglu, M., Tarimcilar, G.,Kaynak, G. 1999. Essential oils of Mentha species from Northern Turkey. Journal of Essential Oil Research 11(5), 579-588.

- Bernath, J. 2001. "Strategies and recent achievements in selection of medicinal and aromatic plants." International Conference on Medicinal and Aromatic Plants. Possibilities and Limitations of Medicinal and Aromatic Plant, p. 576.

- Bertoli, A., Leonardi, M., Krzyzanowska, J., Oleszek, W., Pistelli, L. 2011. Mentha longifolia in vitro cultures as safe source of flavouring ingredients. Acta Biochimica Polonica, 58(4), 581-587.

- Canter, P. H., Thomas, H., Ernst, E. 2005. Bringing medicinal plants into cultivation: opportunities and challenges for biotechnology. Trends in Biotechnology 23(4), 180-185.

- Chaintreau, A. 2001. Simultaneous distillation-extraction: from birth to maturity-review. Flavour and Fragrance Journal 16, 136-148.

- Džamić, A.M.., Soković, M.D., Ristić, M.S., Novaković, M., Grujić-Jovanović, S., Tešević, V., Marin, P.D. 2010. Antifungal and antioxidant activity of Mentha longifolia (L.) Hudson (Lamiaceae) essential oil. Botanica Serbica 34(1), 57-61.

- Figueiredo, C., Barroso, J. G., Pedro, L. G., Scheffer, J. J. C. 2008. 'Factors Affecting Secondary Metabolite Production in Plants: Volatile Components and Essential Oils', Flavour Fragrance Journal 23, 213-226.

- Fleisher, A.,Fleisher, Z. 1998. Volatile extracts of Mentha longifolia growing in Israel. Aromatic Plants of the Holy Land and the Sinai. Part XIII. Journal of Essential Oil Research 10, 647-648.

- Franz, C. M. 2010. Essential oil research: past, present and future. Flavour and FragranceJournal 25(3), 112-113.

- Golparvar. A. R., Hadipanah, A.,Gheisari, M. M. 2013. Chemical analysis and Identification of the components of two ecotypes of Mentha Longifolia L. in Iran province. International Journal of Agriculture and Crop Sciences 5(17), 1946.

- Gulluce, M., Sahin, F., Sokmen, M., Ozer, H, Daferera, D., Sokmen, A., Ozkan, H. 2007. Antimicrobial and antioxidant properties of the essential oils and methanol extract from Mentha longifolia L. ssp. longifolia. Food Chemistry 103(4), 1449-1456. 
- Gupta, A. K., Mishra, R., Singh, A. K., Srivastava, A., Lal, R. K. 2017. Genetic variability and correlations of essential oil yield with agro-economic traits in Mentha species and identification of promising cultivars. Industrial crops and products 95, 726-732.

- Hajlaoui, H., Snoussi, M., Jannet, H.B., Mighri, Z., Bakhrouf, A. 2008. Comparison of chemical composition and antimicrobial activities of Mentha longifolia L. ssp. longifolia essential oil from two Tunisian localities (Gabes and Sidi Bouzid). Annals of Microbiology 58(3), 513-520.

- Hajlaoui, H., Trabelsi, N., Noumi, E., Snoussi, M., Fallah, H., Ksouri, R. and Bakhrouf, A. 2009. Biological activities of the essential oils and methanol extract of tow cultivated mint species (Mentha longifolia and Mentha pulegium) used in the Tunisian folkloric medicine. World Journal of Microbiology and Biotechnology 25(12), 2227-2238.

- Hawrył, M., , Świeboda, R., Hawrył, A., Niemiec, M., Stępak, K., Waksmundzka-Hajnos, M., Szymczak, G. 2015. Micro Two-Dimensional Thin-Layer Chromatography and Chemometric Analysis of Essential Oils from Selected Mentha Species and Its Application in Herbal Fingerprinting. Journal of Liquid Chromatography \& Related Technologies 38, 1794-1801.

- Hussain, A., Anwar, F., Nigam, P.S., Ashraf, M.,Gilani, AH. 2010. Seasonal variation in content, chemical composition and antimicrobial and cytotoxic activities of essential oils from four Mentha species. Journal of the Science of Food and Agriculture 90(11), 1827-1836.

- Jamzad, M., Jamzad, Z., Mokhber, F., Ziareh, S.,Yari, M. 2013. Variation in essential oil composition of Mentha longifolia var. chlorodichtya Rech. f. and Ziziphora clinopodiodes Lam. growing in different habitats. Journal of Medicinal Plants Research 7(22), 1618-1623.

- Kokkini, S. and Papageorgiou, VP. 1988. Constituents of essential oils from Mentha longifolia growing wild in Greece. Planta Medica 54(1), 59-60.

- Kokkini, S., Karousou, R., Lanaras, T. 1995. Essential oils of spearmint (Carvone-rich) plants from the island of Crete (Greece). Biochemical Systematics and Ecology, 23(4), 425-430.

- Koliopoulos, G., Pitarokili, D., Kioulos, E., Michaelakis, A. and Tzakou, O. 2010. Chemical composition and larvicidal evaluation of Mentha, Salvia, and Melissa essential oils against the West Nile virus mosquito Culex pipiens. Parasitology Research 107(2), 327-335.

- Lange, D. 2002. Medicinal and aromatic plants: trade, production, and management of botanical resources. In XXVI International Horticultural Congress: The Future for Medicinal and Aromatic Plants 629 (pp. 177-197).

- Lawrence, BM. 2006. Oil Composition of the Other Mentha Species and Hybrids, B. M. Lawrence, in Mint: The genus Mentha. Florida, USA: CRC Press.

- Llorens-Molina, J.A., Castell, V., Vacas, S. and Verdeguer, M. (2017) TLC-GC/MS method for identifying and selecting valuable essential oil chemotypes from wild populations of Mentha longifolia $\mathrm{L}$ . Natural Volatile and Essential Oils 4 (4), 49-61.

- Lubbe, A., Verpoorte, R. 2011. Cultivation of medicinal and aromatic plants for specialty industrial materials. Industrial crops and products 34(1), 785-801.

- Matovic M.M., Lavadinovlc V. (1999). Essential oil composition of Mentha longifolia (L.) Huds. from the Mountain Zlatar in Yugoslavia. Journal of Essential Oil Products 2, 78-81.

- Maffei, M. 1988. A chemotype of Mentha longifolia (L.) Hudson particularly rich in piperitenone oxide. Flavour and Fragrance Journal 3(1), 23-26.

- Mastelic, J., Jerkovic, I. 2002. Free and glycosidically bound volatiles of Mentha longifolia growing in Croatia. Chemistry of Natural Compounds 38(6), 561-564.

- Mkaddem, M., Bouajila, J., Ennajar, M., Lebrihi, A., Mathieu, F.,Romdhane, M. 2009. Chemical composition and antimicrobial and antioxidant activities of Mentha (longifolia L. and viridis) essential oils. Journal of Food Science 74(7), 358-363.

- Moradalizadeh, M., Khodashenas, M., Amirseifadini, L.,Ganjehkaviri, M. 2014. Identification of chemical compounds in essential oils from stems, leaves and flowers of Mentha longifolia Var. kermanensis by GC/MS. International Journal of Biosciences 4(6), 117-121. 
- Nemeth, É. 2015Natural Variability of Essential Oil Components, in:Handbook of Essential Oils: Science, Technology, and Applications, Second Edition, CRC Press, Boca Raton, Florida, USA.

- Pothier, J., Galand, N., El Ouali, M., Viel, C. 2001.Comparison of planar chromatographic methods (TLC, OPLC, AMD) applied to essential oils of wild thyme and seven chemotypes of thyme." Il Farmaco 56(57), 505-511.

- Saeidi, Z., Saeidi, KA., Salehi, A., Jouneghani, RS., Amirshekari, H. and Taghipour, A. 2012. Essential oil content and composition of Mentha longifolia (L.) Hudson grown wild in Iran. Journal of Medicinal Plants Research 6(29), 4522-4525.

- Salman. M., Abdel-Hameed, E. S.,Bazaid, S., Dabi, M.M. 2015. Chemical composition for hydrodistillation essential oil of Mentha longifolia by gas chromatography-mass spectrometry from north regions in Kingdom of Saudi Arabia. Pharmaceutical Chemistry Journal 7, 34-40.

- Schippmann, U.W.E., Leaman, D.,Cunningham, A.B. 2006. A comparison of cultivation and wild collection of medicinal and aromatic plants under sustainability aspects, in: R. J. Bogers, L.E. Craker \& D. Lange (Eds.). Medicinal and Aromatic Plants, Springer, the Netherlands, pp. 75-95.

- Schippmann, U., Leaman, D.J., Cunningham, A.B. 2002. Impact of cultivation and gathering of medicinal plants on biodiversity: global trends and issues. Biodiversity and the ecosystem approach in agriculture, forestry and fisheries. Inter-Department Working Group on Biology Diversity for Food and Agriculture, FAO, Rome.

- Segev, D., Nitzan, N., Chaimovitsh, D., Eshel, A. and Dudai, N. 2012. Chemical and Morphological Diversity in Wild Populations of Mentha longifolia in Israel. Chemistry \& Biodiversity 9(3), 577-588.

- Sharopov, F.S., Sulaimonova, V.A., Setzer, W.N. 2012. Essential oil composition of Mentha longifolia from wild populations growing in Tajikistan. Journal of Medicinally Active Plants 1(2), 76-84. 